\title{
Early maternal separation is not associated with changes in telomere length in domestic kittens (Felis catus)
}

\author{
Mikel Delgado ${ }^{\text {Corresp., } 1}$, C A Tony Buffington ${ }^{1}$, Melissa Bain ${ }^{1}$, Dana L. Smith ${ }^{2}$, Karen Vernau ${ }^{3}$ \\ ${ }^{1}$ Department of Medicine and Epidemiology, School of Veterinary Medicine, University of California, Davis, Davis, CA, United States \\ Department of Biochemistry and Biophysics, University of California, San Francisco, San Francisco, CA, United States \\ 3 Department of Surgical and Radiological Sciences, School of Veterinary Medicine, University of California, Davis, Davis, CA, United States \\ Corresponding Author: Mikel Delgado \\ Email address: mmdelgado@ucdavis.edu
}

Objective Studies of multiple species have found that adverse early life experiences, including childhood trauma and maternal separation, can result in accelerated telomere shortening. The objective of this study was to determine if premature separation from the mother affected telomere length in domestic kittens (Felis catus). Subjects were 42 orphaned kittens and 10 mother-reared kittens from local animal rescue groups and shelters. DNA was extracted from whole blood collected from kittens at approximately 1 week and 2 months of age. Telomere length was assessed by qPCR (quantitative polymerase chain reaction) from a total of 86 samples and expressed as a ratio of telomere PCR relative to a single copy gene PCR (T/S).

Results A generalized linear mixed model found there were no detectable differences in telomere length based on survival $\left(F_{1,76.2}=3.35, p=0.07\right)$, orphan status $\left(F_{1,56.5}=0.44, p=0.51\right)$, time point $\left(F_{1,43.5}=\right.$ $0.19, p=0.67)$, or the interaction between orphan status and time $\left(F_{1,43.5}=0.86, p=0.36\right)$. Although in other species, telomere shortening is commonly associated with aging, even early in life, we did not find evidence for telomere shortening by two months of age. Our results suggest that the experience of early maternal separation in domestic cats who are subsequently hand-reared by humans does not accelerate telomere shortening compared to mother-reared kittens, at least in the first few months of life. 
1 Early maternal separation is not associated with changes in telomere length in domestic kittens

2 (Felis catus)

3

4 Mikel M. Delgado, $\mathrm{PhD}^{1}$

5 C.A. Tony Buffington, DVM ${ }^{1}$

6 Melissa J. Bain, DVM ${ }^{1}$

7 Dana L. Smith, $\mathrm{PhD}^{2}$

8 Karen M. Vernau, DVM ${ }^{3}$

$9{ }^{1}$ Department of Medicine and Epidemiology, One Shields Avenue, School of Veterinary

10 Medicine, University of California-Davis, Davis, CA 95616

$11{ }^{2}$ Department of Biochemistry and Biophysics, University of California, San Francisco,

12 CA, USA 94158

$13{ }^{3}$ Department of Surgical and Radiological Sciences, One Shields Avenue, School of Veterinary

14 Medicine, University of California-Davis, Davis, CA 95616

15

16 Corresponding author: Mikel M. Delgado

17 mmdelgado@ucdavis.edu 
18 Abstract

19 Objective

20 Studies of multiple species have found that adverse early life experiences, including childhood

21 trauma and maternal separation, can result in accelerated telomere shortening. The objective of

22 this study was to determine if premature separation from the mother affected telomere length in

23 domestic kittens (Felis catus). Subjects were 42 orphaned kittens and 10 mother-reared kittens

24 from local animal rescue groups and shelters. DNA was extracted from whole blood collected

25 from kittens at approximately 1 week and 2 months of age. Telomere length was assessed by

26 qPCR (quantitative polymerase chain reaction) from a total of 86 samples and expressed as a

27 ratio of telomere PCR relative to a single copy gene PCR (T/S).

\section{Results}

29 A generalized linear mixed model found there were no detectable differences in telomere length

30 based on survival $\left(F_{1,76.2}=3.35, p=0.07\right)$, orphan status $\left(F_{1,56.5}=0.44, p=0.51\right)$, time point

$31\left(F_{1,43.5}=0.19, p=0.67\right)$, or the interaction between orphan status and time $\left(F_{1,43.5}=0.86, p=\right.$

32 0.36). Although in other species, telomere shortening is commonly associated with aging, even

33 early in life, we did not find evidence for telomere shortening by two months of age. Our results

34 suggest that the experience of early maternal separation in domestic cats who are subsequently

35 hand-reared by humans does not accelerate telomere shortening compared to mother-reared

36 kittens, at least in the first few months of life.

37

38 Keywords: telomeres, biological aging, domestic cats, maternal separation, telomere attrition 


\section{Introduction}

Early life experiences can impact mental and physical health across the lifespan (Felitti et al. 1998). In particular, adverse childhood experiences (ACEs) have received significant attention in the medical and psychological literature because of the potential harm they can cause, including increased risk for future alcohol and drug abuse, depression, and poor health outcomes (Felitti et al. 1998). In humans and rodents, inadequate maternal care leads to increased fearfulness and negative affect (Hane \& Fox 2006), stereotypies (Latham \& Mason 2008), increased fear and panic in adults (Caldji et al. 1998; Nishi et al. 2014), and decreased cognitive abilities, including spatial navigation (Liu et al. 2000) and memory (Zalosnik et al. 2014).

Studies in multiple species have found that adverse early life experiences and other stressors can also result in accelerated telomere shortening (Chatelain et al. 2020; Cram et al. 2017; Gil et al. 2019; Nettle et al. 2017; Price et al. 2013; Xavier et al. 2018). Telomeres are highly

conserved, non-coding regions of repetitive nucleotide sequences at each end of a chromosome that protect the genome during cell replication by preventing genes from truncating. Cells have a limited reproductive capacity, referred to as the "Hayflick Limit" (Hayflick \& Moorhead 1961). Telomeres shorten during mitosis and once they reach a threshold the cell stops reproducing and enters a state of senescence or dies (Allsopp et al. 1995). Because of this process, telomeres also are associated with aging and the remaining capacity of a cell to replicate (Aubert \& Lansdorp 2008; Chiu \& Harley 1997). Telomeres are subject to more shortening early in life (Frenck et al. 1998), and telomere length is a strong predictor of lifespan and mortality in multiple species (Bize et al. 2009; Cram et al. 2017; Heidinger et al. 2012). Thus, adverse experiences early in life may have more influence on telomere length and long-term health and life-history than do adverse experiences later in life (Ridout et al. 2017). 
62

63

64

Previous studies have demonstrated the effects of adverse early life experiences on telomere length. Among European starlings, an early life competitive disadvantage (being smaller than other brood members) led to more rapid telomere attrition by 12 days of age compared to birds who were larger than the rest of their brood (Nettle et al. 2015). Meerkat pups born into larger groups of young have shorter telomeres due to increased competition for access to milk (Cram et al. 2017). Maternal stress also can shorten telomeres. For example, an increased rate of telomere loss was found in female offspring of laying zebra finches treated with corticosterone compared to controls and the offspring of zebra finches treated with 17- $\beta$-estradiol (Tissier et al. 2014). Social isolation shortens telomeres (Aydinonat et al. 2014), but there have been few studies of the effects of maternal presence or care on telomere length. Children in rural areas of China who were cared for by extended family had shorter telomeres compared to children raised with their mothers (Chen et al. 2019), and individuals who described their parents as neglectful had shorter telomeres than those who did not (Enokido et al. 2014). Similar effects are found in nonhumans; Welsh pony foals that were abruptly weaned and separated from their mothers showed higher levels of stress and shorter telomeres compared to foals who were progressively weaned (Lansade et al. 2018). Rhesus macaques reared by their mothers had longer telomeres than monkeys who were raised with peers or in isolation (Schneper et al. 2016).

Kittens have frequent interactions and physical contact with their mother during the nursing and weaning periods, which typically last until at least two months of age (Albonetti 1988).

Some kittens become inadvertently separated from their mothers before weaning and are subsequently hand-reared by humans. Hand-raising of kittens has become increasingly common as animal shelters improve their ability to care for orphaned neonatal kittens. Orphaned neonatal kittens have a high rate of mortality (Little 2013), are at risk for abnormal behaviors (Delgado et 
85 al. 2020), and show increased distress vocalizations and activity during a nest separation (Lowell

86 et al. 2020), compared to mother-reared kittens. To better understand the effects of early

87 maternal separation on domestic cats, the purpose of the described study was to investigate

88 whether premature separation from the mother affected telomere length in kittens at two time

89 points.

90 Previous studies in adult cats have established a relationship between telomere length and

91 aging (McKevitt et al. 2003), and disease processes such as kidney failure (Quimby et al. 2013).

92 These and other studies have established the feasibility of measuring telomere length in cats

93 (Brümmendorf et al. 2002; Pang \& Argyle 2009). To our knowledge, this is the first study

94 investigating the effect of early maternal separation on telomere length and telomere dynamics in 95 kittens.

96 If maternal separation has deleterious effects, orphaned kittens could show shorter telomeres

97 compared to control kittens. We expected similar telomere length in both groups in the first week

98 of life, as all kittens who were separated from their mothers would have experienced the

99 separation recently. We predicted that all kittens would experience some telomere shortening by

100 eight weeks of age due to the normal process of aging, and we specifically hypothesized an

101 interaction effect, such that kittens who were separated from their mothers early in life (orphans)

102 would have significantly shorter telomeres than kittens raised by mothers (mother-reared) at

103 eight weeks of age.

104 Methods

105 All animal procedures were approved by the Animal Care and Use Committee at the

106 University of California, Davis, under Protocol \#20379.

107 Study Animals

Peer) reviewing PDF | (2020:08:52366:4:0:NEW 25 Mar 2021) 
Subjects were 42 orphaned kittens (OR) and 10 mother-reared (MR) kittens from local rescue

109

110

111

112

113

114

115

116

117

118

119

120

121

122

123

124

125

126

127

128

129

groups and shelters. Orphans had been turned into shelters or rescue groups without their mothers by members of the public. All kittens were entered into the study before they were 7 days of age. Age was assessed by an experienced veterinary technician or shelter rescue staff, as determined by known date of birth, or as estimated by presence of umbilical cord, weight, ear position, and degree of eyes opening (Little 2011). Orphaned kittens were raised in foster homes and were housed in incubators set to $80-90^{\circ} \mathrm{F}$, and with relative humidity set to $50-60 \%$ (Little 2013; Peterson 2011). Incubators included a heating pad and bedding. All orphans were fed commercial kitten formula (Breeder's Edge ${ }^{\circledR}$, Revival Animal Health, Orange City, Iowa) until weaned, when they were fed a commercial dry and wet food (Purina Pro-Plan Kitten Formula ${ }^{\circledR}$, St. Louis, MO). Mother-reared kittens were also cared for in foster homes; they consumed their mother's milk until weaned onto the same type of wet and dry kitten food as the orphans received. Foster caretakers gave written consent for fostered kittens to participate in the study. Nine OR kittens died while in foster (average age of death: 20 days, SD: 11.9 days, range 6 to 42 days); the remaining kittens were adopted into homes after being weaned and neutered.

\section{Experimental Procedures}

Blood $(200 \mu \mathrm{l})$ was drawn from each kitten via jugular venipuncture by a veterinarian or veterinary technician when kittens were approximately 1 week and two months of age (mean age of first blood sample: OR: 8 days, SD: 2 days, MR: 10 days, SD: 1.2 days, range 6-13 days; mean age of second blood sample: OR: 69 days, SD: 9.3 days, MR: 59 days, SD: 4.3 days, range 50-93 days). Blood samples were placed into EDTA tubes then transferred to a cryotube and stored at $-80^{\circ} \mathrm{C}$ until analysis.

Peer] reviewing PDF | (2020:08:52366:4:0:NEW 25 Mar 2021) 
A total of 96 blood samples were collected, and 86 samples were used in the final analysis.

Blood from 28 OR and six MR kittens was collected and analyzed at both time points. Only one

\section{Quantification of telomere length}

The telomere length measurement assay was adapted from Cawthon (Cawthon 2002; Park et

maximum change in slope of the fluorescence amplification curve becomes the crossing point.

The ' $\mathrm{T}$ ' qPCR reaction and ' $\mathrm{S}$ ' qPCR reactions were carried out sequentially. and were considered free of contaminants. 
Whole blood was treated with a 'cell lysis' solution (Qiagen, QIAmp DNA blood mini kit,

154

155

156

157

158

159

160

161

162

163

164

165

166

167

168

169

170

171

172

173

174

175

\#51106) and the lysate was spun through a column that retained the DNA. Subsequent washing and elution released the purified DNA from the column. The DNA extracted from blood from each animal was diluted to $10 \mathrm{ng} / \mathrm{ul}$ in a $96-$ well stock plate. A liquid handler was used to pipet triplicate reactions from the 96-well DNA plate to a 384-well ' $\mathrm{T}$ ' (telomere) reaction plate, each well containing $7.5 \mathrm{ul}$ of PCR reaction mixture to give $2.5 \mathrm{ng} / \mathrm{ul}$ final DNA concentration. Immediately after the T reactions were put into the PCR machine, the same DNA stocks were used to pipet triplicate reactions on a separate 384-well 'S' (single copy gene) reaction plate.

For the feline single copy gene (S), primers were made to amplify feline GAPDH. For the forward primer, we used the sequence (5'->3') GTGGTGAAGCAGGCATCAGA and for the reverse primer, we used CACTGTTAAAGTCGCAGGAGACA. Both primers were used at a concentration of $1 \mu \mathrm{M}$ in the PCR reaction. The primers used for the telomere (T) PCR were Tel C [5'-TGTTAGGTATCCCTATCCCTATC-3'], at a final concentration of $200 \mathrm{nM}$, and Tel G [5'-ACACTAAGGTTTGGGTTTGGGTT-3'], at a final concentration of $400 \mathrm{nM}$.

Both telomere and single copy gene PCR reactions were performed with QuantiFast SYBR Green PCR Kit (QIAGEN) on a Roche LightCycler 480 Realtime PCR machine (LC480; Roche Diagnostics Corporation, Indianapolis, IN). T and S reactions were 10ul with stock 2x Quantifast mix (5ul/reaction). The Quantifast mix contains a proprietary fluorescent polymerase for PCR. The concentration of DNA in all experimental reactions was $2.5 \mathrm{ng} / \mathrm{ul}$.

The thermal PCR cycling profiles were as follows: for T (telomeric), denature at $95^{\circ} \mathrm{C}$ for 15 minutes, one cycle; denature at $94^{\circ} \mathrm{C}$ for 15 seconds, anneal/extend at $49^{\circ} \mathrm{C}$ for 15 seconds, one cycle. Denature at $94^{\circ}$ for 15 seconds, anneal/extend at $49^{\circ} \mathrm{C}$ for 30 seconds, one cycle. Melt at $95 \mathrm{C}^{\circ}$ for $15 \mathrm{~seconds}$, anneal at $62^{\circ} \mathrm{C}$ for $10 \mathrm{~seconds}, 74^{\circ} \mathrm{C}$ for 15 seconds with fluorescence data 
176 collection 32 cycles. For $\mathrm{S}$ (single copy gene), denature at $95^{\circ} \mathrm{C}$ for 10 minutes, one cycle;

177 denature at $94^{\circ} \mathrm{C}$ for 15 seconds, anneal at $60^{\circ} \mathrm{C}$ for 60 seconds with fluorescence data collection,

17845 cycles. A standard curve was created from two-fold serial dilutions of pooled genomic DNA

179 from four OR kittens, from which the concentrations of telomere reactions and single copy gene

180 reactions were determined. A 5-point, 2x-dilution standard curve was made of pooled genomic

181 DNA from four OR kittens, starting with 10ng/ul, and ending with .312ng/ul. At least three runs

182 (T and S paired runs) were carried out for each sample. The efficiency of every T run was greater

183 than $90 \%$ and the efficiency of every $\mathrm{S}$ run was greater than $95 \%$. The $\mathrm{R}^{2}$ For $\mathrm{T} 1, \mathrm{~S} 1, \mathrm{~T} 2$, and $\mathrm{S} 2$

184 runs were $1.0, .99, .99$, and 1.0 respectively.

185 Genomic DNAs from the same 4 kittens were plated individually as positive controls, to

186 serve as quality controls for each run. Three pairs of T and S PCR reactions were run on different

187 days and the T/S PCR ratio was calculated for each pair. If, after three runs, a value looked like

188 an outlier, it was discarded and an additional run was made. For this plate of samples, the inter-

189 plate CV (the standard deviation/average of the $2 \mathrm{~T} / \mathrm{S}$ values) for every sample was averaged

190 and came to $3 \%$.

191 Statistical analyses

192 All data were analyzed using SAS University Edition (SAS Institute Inc, Cary, NC). To

193 assess the effect of orphan status and age on telomere length, we used a linear mixed model

194 (LMM) in PROC MIXED including survival (Y/N), orphan status (Y/N), time point (1 or 2), and

195 the interaction between orphan status and time point as predictors, with telomere ratio as the

196 dependent variable. The residuals of the LMM met the assumptions of normality (q-q plot) and

197 homogeneity (Levene's test, $p>0.05$ ), and data from all groups also met the assumption of

198 equality of variance (all Satterthwaite tests, $p>0.05$ ). Litter and kitten ID (nested within litter) 
199 were included as random effects to account for repeated measures of the same individuals and

200 the effects of relatedness on kittens from the same litter. Telomere lengths followed a normal

201 distribution (Kolmogorov-Smirnov test $=0.07, p>0.15$ ) and we used a Pearson's correlation to

202 assess the relationship between telomere lengths at time points 1 and 2 for kittens who had been

203 sampled twice. We also performed a post-hoc sensitivity analysis in G*Power 3.1.9.2 (University

204 of Kiel, Germany; Faul et al. 2007) to determine the effect size that would have been necessary

205 to find a difference between groups. Figures were created in R 4.0.2 (R Foundation for Statistical

206 Computing, Vienna, Austria).

207 Results

208 Telomere ratios ranged from 0.63 to $1.44(x=0.95, S D=0.14)$. The model found no effect of 209 survival $\left(F_{1,76.2}=3.35, p=0.07\right)$, orphan status $\left(F_{1,56.5}=0.44, p=0.51\right)$, time point $\left(F_{1,43.5}=\right.$

$2100.19, p=0.67)$, or the interaction between orphan status and time point $\left(F_{1,43.5}=0.86, p=0.36\right)$;

211 Figure 1). The results were the same when assessing only the 34 kittens from whom we had two

212 blood samples (orphan status $\left(F_{1,45.4}=0.75, p=0.39\right)$, time point $\left(F_{1,32}=0.01, p=0.91\right)$, or the

213 interaction between orphan status and time point $\left.\left(F_{1,32}=0.72, p=0.40\right)\right)$.

214 There was a small but statistically significant correlation between telomere length at time 1

215 and time $2, r(34)=0.36, p=0.04$; Figure 2 . When comparing by orphan status, the correlation

216 for MR kittens was $r(6)=0.80(p=0.06)$, and for OR kittens was $r(28)=0.28(p=0.14)$,

217 neither of which was statistically significant.

218 Sensitivity analysis

219 Because of the null findings between groups, the post-hoc assessment of the sensitivity of the 220 analysis allowed us to determine the effect size that would have been necessary to find a

221 difference between groups. With adequate power $(\beta=0.80, \alpha=0.05)$, to find a statistically 
222 significant difference between the number of OR and MR kittens we examined at each time point

223 would have required a large effect size (Cohen's $d=1.11$ and 0.88 respectively). This would

224 have equaled a difference in $\mathrm{T} / \mathrm{S}$ ratio between groups of at least 0.13 for Time 1 and 0.14 for

225 Time 2. For the pooled data for all kittens, to find a difference between the two time points

226 would have required a medium effect size of Cohen's $d=0.54$, which would have equaled a

227 difference between groups in $\mathrm{T} / \mathrm{S}$ ratio of at least 0.08 . The means of both groups were very

228 close (0.98 and .97 for Time 1; 0.98 and 0.93 in Time 2), and the differences resulted in effects

229 sizes of 0.07 (Time 1) and 0.35 (Time 2). If our findings came from representative samples, we

230 would have needed samples from more than 5000 and 200 kittens, respectively, to identify a

231 statistically significant effect at either time point.

\section{Discussion}

233 We found no detectable differences in telomere length between orphaned and mother-reared

234 kittens. We predicted that by approximately two months, orphaned kittens would have shorter

235 telomeres than mother-reared kittens and would experience more telomere attrition. Although

236 telomere shortening is commonly associated with aging, we also did not find evidence for

237 telomere shortening in either group of kittens between the first week of life and approximately

238 two months later.

239 Some previous studies have found deleterious effects of early maternal separation, including 240 telomere shortening, in other species (Chen et al. 2019; Enokido et al. 2014; Lansade et al. 2018;

241 Schneper et al. 2016). Previous studies have found that being orphaned increases behavioral

242 signs of stress and other abnormal behaviors compared to mother-reared kittens (Delgado et al.

243 2020; Lowell et al. 2020). However, for domestic cats, being raised by humans in the absence of

244 the mother may also have some protective effects. Kittens are altricial and unlikely to survive 
245 when orphaned without outside care. Being raised by humans may be more like progressive

246 weaning; foals who were gradually weaned experienced less telomere shortening than abruptly

247 weaned foals (Lansade et al. 2018). All of the orphans in our study were maintained at consistent

248 temperatures and humidity and were bottle-fed, which may have lowered stress and prevented

249 competition for food as is experienced in many young animals (Hudson \& Trillmich 2008),

250 possibly preventing telomere loss. Further research is needed to better understand whether

251 human care can compensate for the impact of maternal loss in kittens.

252 Alternatively, it is possible that the mothers in our study were under an increased stress level, 253 thereby shortening the telomeres of the mother-reared kittens. This explanation seems unlikely as

254 all mother cats were social with humans, and housed in quiet, private, indoor environments.

255 Instead, it appears that any potential stress in cats related to being orphaned does not have a large

256 impact on telomere length. Cortisol reduces the production of telomerase (Choi et al. 2008), an

257 enzyme which may preserve telomere length (Boccardi \& Paolisso 2014). Studies of stress in

258 adult cats have not consistently found a strong correlation between cortisol and other signs of

259 stress (Fukimoto et al. 2020; Gourkow et al. 2014), suggesting one potential explanation for why

260 orphaned kittens may show more behavioral signs of stress but no telomere shortening compared

261 to mother-reared kittens. Further studies are needed to explore whether stress increases cortisol

262 level in neonatal kittens.

263 A longitudinal approach is useful for assessing the effects of aging on telomere length. We

264 did not track kittens into adulthood; ideally, measurements of survival and telomere length would

265 be taken from cats at multiple time points to assess whether premature maternal separation has

266 any long-term effects on health. Among kittens who survived to two months of age (100\% of

267 mothered kittens and $79 \%$ of all orphans), we found no evidence of an impact of being orphaned 
268 on telomere length. It is possible that our inability to find signs of telomere shortening in either

269 study group may have been due to the short time interval between sample collection

270 (approximately 8 weeks). Longitudinal studies of young birds, (Nettle et al. 2017; Nettle et al.

271 2015) sheep (Fairlie et al. 2016), adult laboratory mice (Cai et al. 2015), and Sudanian grass rats

272 (Grosbellet et al. 2015) found significant shortening of telomeres within similar or smaller time

273 frames (Bateson 2016). However, we cannot know if rates of attrition across species are

274 comparable.

275 Rather than finding telomere shortening, we found a correlation between relative telomere

276 length at each time point. Kittens with relatively shorter telomeres at time 1 also had relatively

277 shorter telomeres at time 2. Some orphans appeared to have increased relative telomere length,

278 whereas a decreased relative telomere length was found in others, suggesting more variability

279 among orphans.

280 Our study had some limitations. We had difficulty procuring sufficient blood samples from

281 mother-reared kittens, leaving us with imbalanced groups and power sufficient only to detect

282 medium to large effect sizes. Our sample may also be biased by the fact that we could not re-test

283 nine kittens (all orphans) who died after their initial blood sample was collected. Our model

284 suggested that the initial telomere lengths of kittens who died were not different from the rest of

285 the population sampled, and we did not find any differences between surviving orphans and

286 mother-reared kittens. Finally, there are different ways to assess telomere length, including

287 qPCR, Q-FISH, TRF analysis, and TeSLA (Lai et al. 2018; Montpetit et al. 2014). qPCR offers

288 the benefits of being easy to perform and requiring small amounts of DNA. However, qPCR can

289 only provide relative, rather than absolute telomere length, and is less sensitive to short telomeres 
290 or telomeropathies (Gutierrez-Rodrigues et al. 2014; Lai et al. 2018). Thus, using qPCR may

291 have reduced our ability to find differences between our two populations of interest.

292 Conclusions

293 Although there may be some reported deleterious effects of early maternal separation in 294 domestic cats (Ahola et al. 2017; Delgado et al. 2020; Lowell et al. 2020), we did not find that 295 orphaned kittens had shorter telomeres than non-orphans at approximately one week or two 296 months of age. If differences do exist between the two groups, they are likely small and of 297 unknown clinical significance. Further research, ideally with larger samples, should assess 298 whether there are differences between orphaned and mother-reared populations later in life. 299

300 Abbreviations

301 MR: mother-reared

302 OR: orphaned

303 PCR: polymerase chain reaction

304 qPCR: quantitative polymerase chain reaction

305 S: single copy gene

306 T: telomere

307

308

Authors' contributions

$\mathrm{MD}, \mathrm{CATB}, \mathrm{MB}$ and $\mathrm{KV}$ designed the study. MD and $\mathrm{KV}$ were responsible for data collection. DS conducted PCR/telomere analyses. MD analyzed the data and was a major contributor in

311 writing the manuscript. All authors read and approved the final manuscript.

\section{Acknowledgements}


313 We thank everyone who assisted with the completion of this research, including: Dr. Jue Lin

314 from the Blackburn Lab at UCSF; Cara Wademan, Samantha Barnum and Emir Hodzic from the 315 UC Davis Veterinary Genetics Laboratory; Dr. Casey Kohen, Meghan Ramczyk, Dallas

316 Butterfield, Emma Hewitt, Robert Collins, Kathy Pinkston, Marcy Vaughn, and Abrah Wymore.

317 We especially thank the foster caretakers and rescue groups who cared for the kittens in this

318 study. We also thank the three reviewers whose constructive feedback helped to improve the 319 manuscript. 
320

321

322

323

324

325

326

327

328

329

330

331

332

333

334

335

336

337

338

339

340

341

342

References

Ahola MK, Vapalahti K, and Lohi H. 2017. Early weaning increases aggression and stereotypic behaviour in cats. Scientific Reports 7:10412. 10.1038/s41598-017-11173-5

Albonetti ME. 1988. Behavioural development in kittens: Effects of litter sex-ratio. Italian Journal of Zoology 22:53-61. 10.1080/00269786.1988.10736541

Allsopp RC, Chang E, Kashefi-Aazam M, Rogaev EI, Piatyszek MA, Shay JW, and Harley CB. 1995. Telomere shortening is associated with cell division in vitro and in vivo. Experimental Cell Research 220:194-200. 10.1006/excr.1995.1306

Aubert G, and Lansdorp PM. 2008. Telomeres and aging. Physiological Reviews 88:557-579. 10.1152/physrev.00026.2007

Aydinonat D, Penn DJ, Smith S, Moodley Y, Hoelzl F, Knauer F, and Schwarzenberger F. 2014. Social isolation shortens telomeres in African Grey parrots (Psittacus erithacus erithacus). PLoS One 9:e93839. 10.1371/journal.pone.0093839

Bateson M. 2016. Cumulative stress in research animals: Telomere attrition as a biomarker in a welfare context? Bioessays 38:201-212. 10.1002/bies.201500127

Bize P, Criscuolo F, Metcalfe NB, Nasir L, and Monaghan P. 2009. Telomere dynamics rather than age predict life expectancy in the wild. Proceedings of the Royal Society B: Biological Sciences 276:1679-1683. doi:10.1098/rspb.2008.1817

Boccardi V, and Paolisso G. 2014. Telomerase activation: A potential key modulator for human healthspan and longevity. Ageing Research Reviews 15:1-5. 10.1016/j.arr.2013.12.006

Brümmendorf TH, Mak J, Sabo KM, Baerlocher GM, Dietz K, Abkowitz JL, and Lansdorp PM. 2002. Longitudinal studies of telomere length in feline blood cells. Experimental Hematology 30:1147-1152. 10.1016/s0301-472x(02)00888-3 
343 Cai N, Chang S, Li Y, Li Q, Hu J, Liang J, Song L, Kretzschmar W, Gan X, Nicod J, Rivera M,

344

345

346

347

348

349

350

351

352

353

354

355

356

357

358

359

360

361

362

363
Deng H, Du B, Li K, Sang W, Gao J, Gao S, Ha B, Ho HY, Hu C, Hu J, Hu Z, Huang G, Jiang G, Jiang T, Jin W, Li G, Li K, Li Y, Li Y, Li Y, Lin YT, Liu L, Liu T, Liu Y, Liu Y, Lu Y, Lv L, Meng H, Qian P, Sang H, Shen J, Shi J, Sun J, Tao M, Wang G, Wang G, Wang J, Wang L, Wang X, Wang X, Yang H, Yang L, Yin Y, Zhang J, Zhang K, Sun N, Zhang W, Zhang X, Zhang Z, Zhong H, Breen G, Wang J, Marchini J, Chen Y, Xu Q, Xu X, Mott R, Huang GJ, Kendler K, and Flint J. 2015. Molecular signatures of major depression. Curr Biol 25:1146-1156. 10.1016/j.cub.2015.03.008

Caldji C, Tannenbaum B, Sharma S, Francis D, Plotsky PM, and Meaney MJ. 1998. Maternal care during infancy regulates the development of neural systems mediating the expression of fearfulness in the rat. Proceedings of the National Academy of Sciences 95:5335-5340.

Cawthon RM. 2002. Telomere measurement by quantitative PCR. Nucleic Acids Research 30:e47-e47.

Chatelain M, Drobniak SM, and Szulkin M. 2020. The association between stressors and telomeres in non-human vertebrates: A meta-analysis. Ecology Letters 23:381-398. $10.1111 /$ ele. 13426

Chen X, Zeng C, Gong C, Zhang L, Wan Y, Tao F, and Sun Y. 2019. Associations between early life parent-child separation and shortened telomere length and psychopathological outcomes during adolescence. Psychoneuroendocrinology 103:195-202. 10.1016/j.psyneuen.2019.01.021 
364 Chiu C-P, and Harley CB. 1997. Replicative senescence and cell immortality: The role of

365 telomeres and telomerase. Proceedings of the Society for Experimental Biology and

366 Medicine 214:99-106. 10.3181/00379727-214-44075

367

368

369

370

371

372

373

374

375

376

377

378

379

380

381

382

383

384

385

386

Choi J, Fauce SR, and Effros RB. 2008. Reduced telomerase activity in human T lymphocytes exposed to cortisol. Brain, Behavior, and Immunity 22:600-605.

$$
\text { 10.1016/j.bbi.2007.12.004 }
$$

Cram DL, Monaghan P, Gillespie R, and Clutton-Brock T. 2017. Effects of early-life competition and maternal nutrition on telomere lengths in wild meerkats. Proceedings of the Royal Society B: Biological Sciences 284. 10.1098/rspb.2017.1383

Delgado MM, Walcher I, and Buffington CAT. 2020. A survey-based assessment of risk factors for cross-sucking behaviors in neonatal kittens, Felis catus. Applied Animal Behaviour Science 230:105069. 10.1016/j.applanim.2020.105069

Enokido M, Suzuki A, Sadahiro R, Matsumoto Y, Kuwahata F, Takahashi N, Goto K, and Otani K. 2014. Parental care influences leukocyte telomere length with gender specificity in parents and offsprings. BMC Psychiatry 14:277. 10.1186/s12888-014-0277-9

Fairlie J, Holland R, Pilkington JG, Pemberton JM, Harrington L, and Nussey DH. 2016. Lifelong leukocyte telomere dynamics and survival in a free-living mammal. Aging Cell 15:140-148. 10.1111/acel.12417

Faul F, Erdfelder E, Lang A-G, and Buchner A. 2007. G* Power 3: A flexible statistical power analysis program for the social, behavioral, and biomedical sciences. Behavior research methods 39:175-191.

Felitti VJ, Anda RF, Nordenberg D, Williamson DF, Spitz AM, Edwards V, Koss MP, and Marks JS. 1998. Relationship of childhood abuse and household dysfunction to many of 
the leading causes of death in adults: The Adverse Childhood Experiences (ACE) Study. American Journal of Preventive Medicine 14:245-258. 10.1016/S0749-3797(98)00017-8

Frenck RW, Blackburn EH, and Shannon KM. 1998. The rate of telomere sequence loss in human leukocytes varies with age. Proceedings of the National Academy of Sciences 95:5607-5610.

Fukimoto N, Melo D, Palme R, Zanella AJ, and Mendonça-Furtado O. 2020. Are cats less stressed in homes than in shelters? A study of personality and faecal cortisol metabolites. Applied Animal Behaviour Science 224:104919. 10.1016/j.applanim.2019.104919

Gil D, Alfonso-Iñiguez S, Pérez-Rodríguez L, Muriel J, and Monclús R. 2019. Harsh conditions during early development influence telomere length in an altricial passerine: Links with oxidative stress and corticosteroids. Journal of evolutionary biology 32:111-125.

Gourkow N, LaVoy A, Dean GA, and Phillips CJC. 2014. Associations of behaviour with secretory immunoglobulin A and cortisol in domestic cats during their first week in an animal shelter. Applied Animal Behaviour Science 150:55-64. 10.1016/j.applanim.2013.11.006

Grosbellet E, Zahn S, Arrivé M, Dumont S, Gourmelen S, Pévet P, Challet E, and Criscuolo F. 2015. Circadian desynchronization triggers premature cellular aging in a diurnal rodent. The FASEB Journal 29:4794-4803. 10.1096/fj.14-266817

Gutierrez-Rodrigues F, Santana-Lemos BA, Scheucher PS, Alves-Paiva RM, and Calado RT. 2014. Direct comparison of flow-FISH and qPCR as diagnostic tests for telomere length measurement in humans. PLoS One 9:e113747.

Hane AA, and Fox NA. 2006. Ordinary variations in maternal caregiving influence human infants' stress reactivity. Psychological science 17:550-556. 
410 Hayflick L, and Moorhead PS. 1961. The serial cultivation of human diploid cell strains.

411

412

413

414

415

416

417

418

419

420

421

422

423

424

425

426

427

428

429

430

431

432
Experimental Cell Research 25:585-621. 10.1016/0014-4827(61)90192-6

Heidinger BJ, Blount JD, Boner W, Griffiths K, Metcalfe NB, and Monaghan P. 2012. Telomere length in early life predicts lifespan. Proceedings of the National Academy of Sciences 109:1743-1748. 10.1073/pnas.1113306109

Hudson R, and Trillmich F. 2008. Sibling competition and cooperation in mammals: Challenges, developments and prospects. Behavioral Ecology and Sociobiology 62:299-307.

Lai T-P, Wright WE, and Shay JW. 2018. Comparison of telomere length measurement methods. Philosophical Transactions of the Royal Society B: Biological Sciences 373:20160451.

Lansade L, Foury A, Reigner F, Vidament M, Guettier E, Bouvet G, Soulet D, Parias C, Ruet A, Mach N, Lévy F, and Moisan M-P. 2018. Progressive habituation to separation alleviates the negative effects of weaning in the mother and foal. Psychoneuroendocrinology 97:5968. 10.1016/j.psyneuen.2018.07.005

Latham NR, and Mason G. 2008. Maternal deprivation and the development of stereotypic behaviour. Applied Animal Behaviour Science 110:84-108.

Little S. 2011. Feline pediatrics: How to treat the small and the sick. Compendium: Continuing Education For Veterinarians 33:1-6.

Little S. 2013. Playing mum: Successful management of orphaned kittens. Journal of Feline Medicine and Surgery 15:201-210. 10.1177/1098612X13477542

Liu D, Diorio J, Day JC, Francis DD, and Meaney MJ. 2000. Maternal care, hippocampal synaptogenesis and cognitive development in rats. Nature Neuroscience 3:799-806.

Lowell KJ, Delgado MM, Mederos SL, and Bain MJ. 2020. The effect of premature maternal separation on distress vocalizations and activity in kittens (Felis catus) during a brief nest

Peer] reviewing PDF | (2020:08:52366:4:0:NEW 25 Mar 2021) 
433

434

435

436

437

438

439

440

441

442

443

444

445

446

447

448

449

450

451

452

453

454

455

separation. Applied Animal Behaviour Science 232:105130.

10.1016/j.applanim.2020.105130

McKevitt TP, Nasir L, Wallis CV, and Argyle DJ. 2003. A cohort study of telomere and telomerase biology in cats. American Journal of Veterinary Research 64:1496-1499. 10.2460/ajvr.2003.64.1496

Montpetit AJ, Alhareeri AA, Montpetit M, Starkweather AR, Elmore LW, Filler K, Mohanraj L, Burton CW, Menzies VS, and Lyon DE. 2014. Telomere length: A review of methods for measurement. Nursing research 63:289.

Nettle D, Andrews C, Reichert S, Bedford T, Kolenda C, Parker C, Martin-Ruiz C, Monaghan P, and Bateson M. 2017. Early-life adversity accelerates cellular ageing and affects adult inflammation: Experimental evidence from the European starling. Scientific Reports 7:40794. 10.1038/srep40794

Nettle D, Monaghan P, Gillespie R, Brilot B, Bedford T, and Bateson M. 2015. An experimental demonstration that early-life competitive disadvantage accelerates telomere loss. Proceedings of the Royal Society B: Biological Sciences 282:20141610.

Nishi M, Horii-Hayashi N, and Sasagawa T. 2014. Effects of early life adverse experiences on the brain: Implications from maternal separation models in rodents. Frontiers in Neuroscience 8:166.

Pang LY, and Argyle DJ. 2009. Using naturally occurring tumours in dogs and cats to study telomerase and cancer stem cell biology. Biochimica et Biophysica Acta (BBA) Molecular Basis of Disease 1792:380-391. 10.1016/j.bbadis.2009.02.010

Park S-J, Kim Y-H, Huh J-W, Lee S-R, Kim S-H, Kim S-U, Kim J-S, Jeong K-J, Kim K-M, Kim H-S, and Chang K-T. 2013. Selection of new appropriate reference genes for RT-qPCR 
456

457

458

459

460

461

462

463

464

465

466

467

468

469

470

471

472

473

474

475

476

477

analysis via transcriptome sequencing of cynomolgus monkeys (Macaca fascicularis). PLoS One 8:e60758-e60758. 10.1371/journal.pone.0060758

Peterson ME. 2011. Care of the orphaned puppy and kitten. In: Peterson M, and Kutzler M, eds. Small Animal Pediatrics. Philadelphia, PA: WB Saunders, 67-72.

Price LH, Kao H-T, Burgers DE, Carpenter LL, and Tyrka AR. 2013. Telomeres and early-life stress: An overview. Biological psychiatry 73:15-23. 10.1016/j.biopsych.2012.06.025

Quimby JM, Maranon DG, Battaglia CL, McLeland SM, Brock WT, and Bailey SM. 2013. Feline chronic kidney disease is associated with shortened telomeres and increased cellular senescence. American Journal of Physiology-Renal Physiology 305:F295-303. 10.1152/ajprenal.00527.2012

Ridout KK, Levandowski M, Ridout SJ, Gantz L, Goonan K, Palermo D, Price LH, and Tyrka AR. 2017. Early life adversity and telomere length: A meta-analysis. Molecular psychiatry. 10.1038/mp.2017.26

Schneper LM, Brooks-Gunn J, Notterman DA, and Suomi SJ. 2016. Early-life experiences and telomere length in adult rhesus monkeys: An exploratory study. Psychosomatic medicine 78:1066-1071. 10.1097/PSY.0000000000000402

Tissier ML, Williams TD, and Criscuolo F. 2014. Maternal effects underlie ageing costs of growth in the zebra finch (Taeniopygia guttata). PLoS One 9:e97705.

10.1371/journal.pone.0097705

Xavier G, Spindola LM, Ota VK, Carvalho CM, Maurya PK, Tempaku PF, Moretti PN, Mazotti DR, Sato JR, and Brietzke E. 2018. Effect of male-specific childhood trauma on telomere length. Journal of psychiatric research 107:104-109. 
478 Zalosnik M, Pollano A, Trujillo V, Suarez M, and Durando P. 2014. Effect of maternal

479 separation and chronic stress on hippocampal-dependent memory in young adult rats:

480 Evidence for the match-mismatch hypothesis. Stress 17:445-450.

481 


\section{Figure Legends}

483 Figure 1. Boxplots of telomere lengths with sample sizes for mother-reared and orphaned 484 kittens at 1 and 8 weeks of age summarized from raw data. Boxplots include median value, IQR 485 (interquartile range), and outliers.

\section{6}

Figure 2. Pearson's correlations between telomere lengths at approximately one week and two months of age for six mother-reared kittens $(r=0.80, p=0.06)$ and 28 orphaned kittens $(r=$ $0.28, p=0.14$ ) for whom repeated samples were collected. The overall correlation for all repeated samples was $r(34)=0.36, p=0.04$. 
Figure 1

Boxplots of telomere lengths with sample sizes for mother-reared and orphaned kittens at 1 and 8 weeks of age summarized from raw data.

Boxplots of telomere lengths with means and sample sizes for mother-reared and orphaned kittens at 1 and 8 weeks of age summarized from raw data. Boxplots include median value, IQR (interquartile range), and outliers.

Telomere Length by Orphan Status at Two Time Points

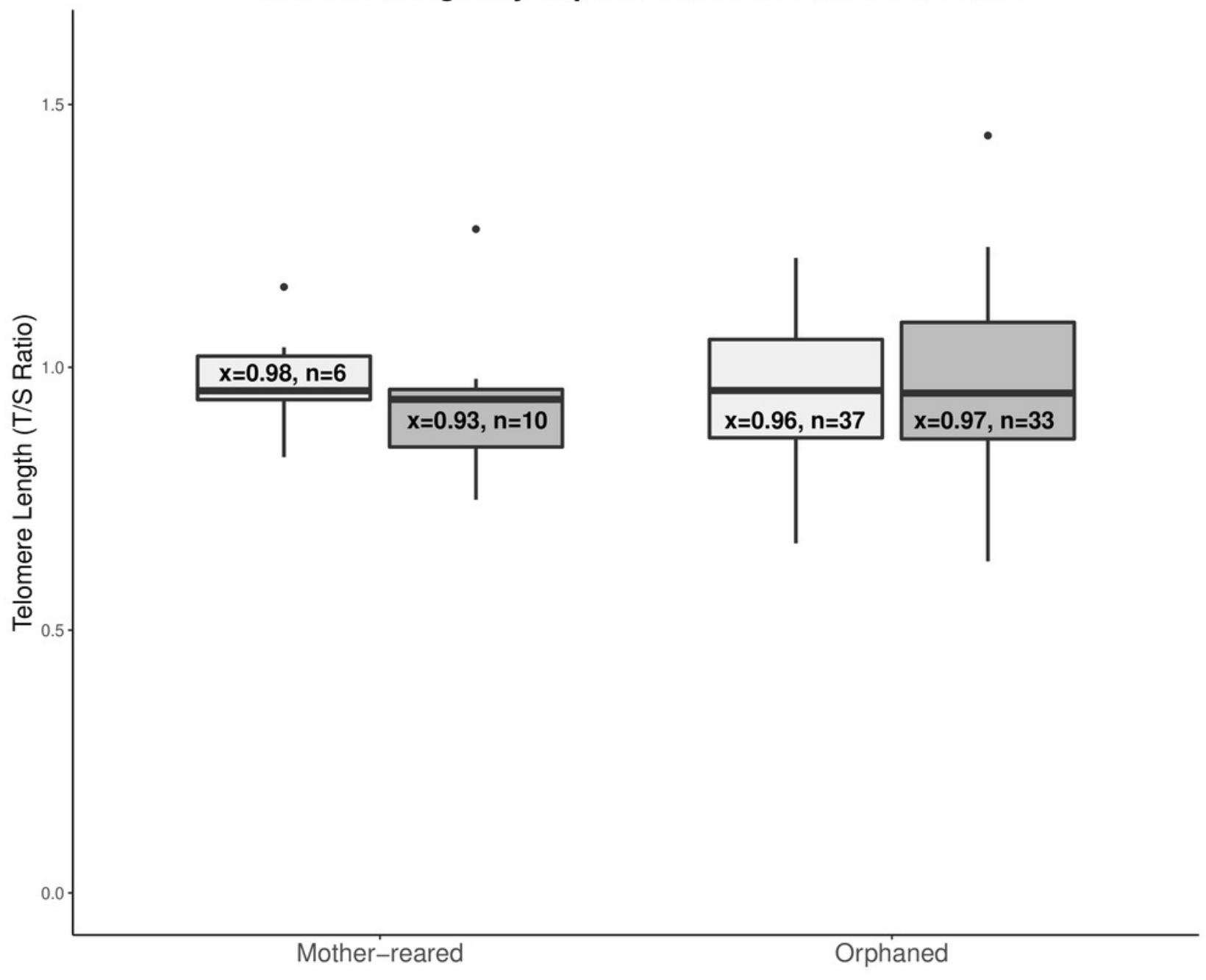


Figure 2

Scatter plot of correlations between telomere lengths at two time points.

Pearson's correlations between telomere lengths at approximately one week and two months of age for six mother-reared kittens $(r=0.80, p=0.06)$ and 28 orphaned kittens $(r=450$ $0.28, p=0.14$ ) for whom repeated samples were collected. The overall correlation for all repeated samples was $r(34)=0.36, p=0.04$.

Telomere Length (T/S Ratio) Correlation at Times 1 and 2

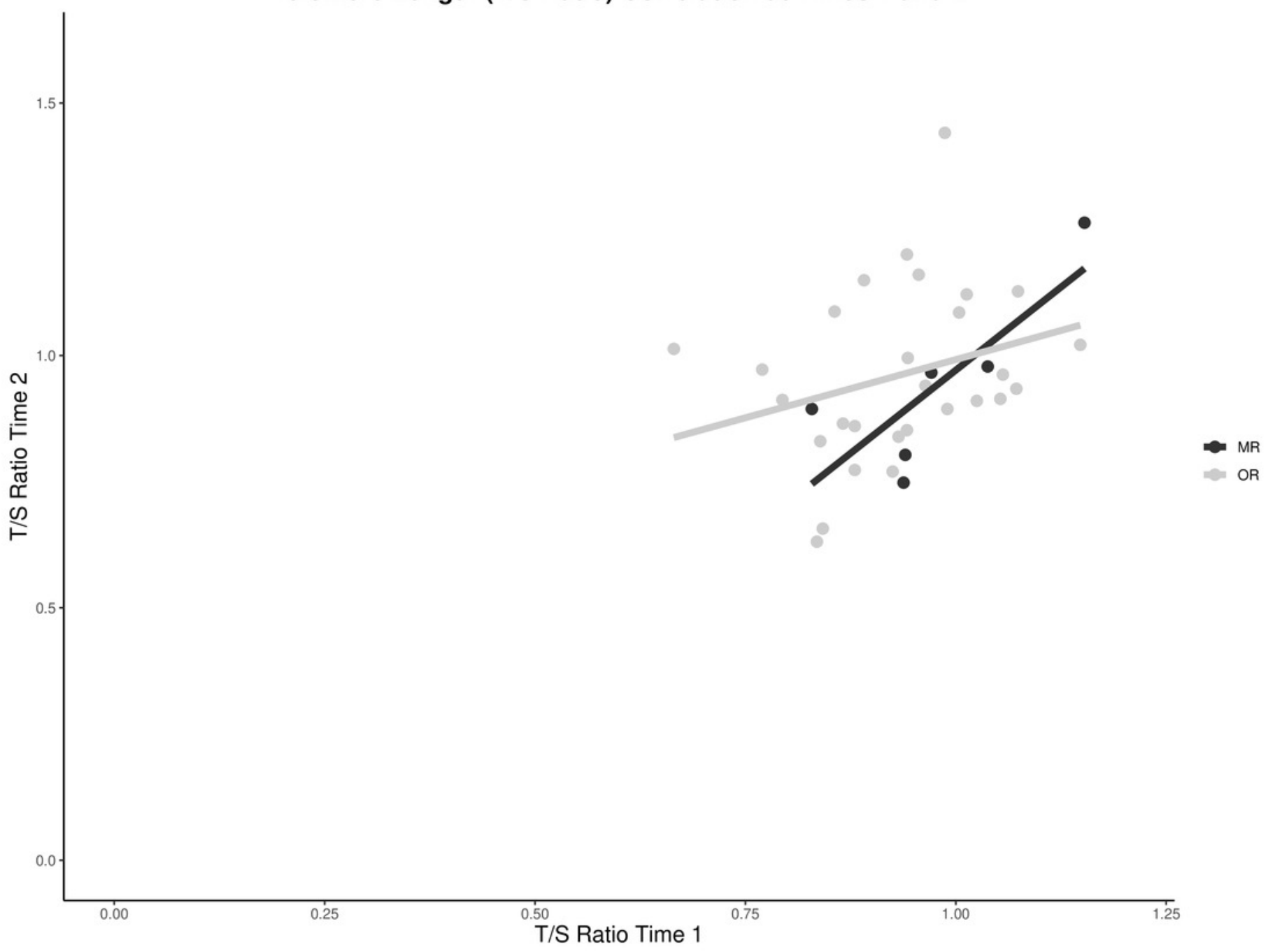




\section{Table $\mathbf{1}$ (on next page)}

Number of blood samples acquired and used in analyses.

Number of blood samples acquired and used in analyses from mother-reared and orphaned kittens at Time 1 (one week) and Time 2 (two months). The number of repeated samples from each group is indicated in lighter text and parentheses. 
1 Table 1. Number of blood samples acquired and used in analyses from mother-reared and orphaned 2 kittens at Time 1 (one week) and Time 2 (two months). The number of repeated samples from each group 3 is indicated in lighter text and parentheses.

\begin{tabular}{llccc}
\hline Source & $\begin{array}{c}\text { Samples not used in } \\
\text { final analyses }\end{array}$ & $\begin{array}{c}\text { Analyzed } \\
\text { samples } \\
\text { Time 1 }\end{array}$ & $\begin{array}{c}\text { Analyzed samples } \\
\text { Time 2 }\end{array}$ & $\begin{array}{c}\text { Total samples } \\
\text { collected }\end{array}$ \\
\hline $\begin{array}{l}\text { Mother-reared } \\
\text { kittens }\end{array}$ & $\begin{array}{l}3 \text { lost due to } \\
\text { experimenter error }\end{array}$ & $6^{\mathrm{a}}$ & $10^{\mathrm{b}}$ & 19 \\
$\begin{array}{l}\text { Repeated } \\
\text { samples }\end{array}$ & $(6)$ & $(6)$ & \\
$\begin{array}{l}\text { Orphaned } \\
\text { kittens }\end{array}$ & 4 assay development & $37^{\mathrm{c}}$ & $33^{\mathrm{d}}$ & 77 \\
$\quad$ Repeated & 3 insufficient DNA & $(28)$ & $(28)$ & \\
samples & & 43 & 43 & 96 \\
\hline
\end{tabular}

4 Note: all ten samples not used in final analyses were acquired from Time 1.

5 asamples from 3 litters

6 b4 litters

7 c16 litters

$8{ }^{\mathrm{d}} 17$ litters 